\title{
Screening of gestational diabetes mellitus: comparison of glucose challenge test done in non fasting state (DIPSI criteria) and fasting state (WHO criteria): a prospective study
}

\author{
Surabhi S. Ardhapurkar, Shailesh J. Kore*, Pradnya A. Supe
}

Department of Obstetrics and Gynecology, LTMMC and LTMGH, Sion, Mumbai, Maharashtra, India

\author{
Received: 03 June 2019 \\ Revised: 28 October 2020 \\ Accepted: 29 October 2020 \\ *Correspondence: \\ Dr. Shailesh J. Kore, \\ E-mail: shaileshkore@hotmail.com
}

Copyright: () the author(s), publisher and licensee Medip Academy. This is an open-access article distributed under the terms of the Creative Commons Attribution Non-Commercial License, which permits unrestricted non-commercial use, distribution, and reproduction in any medium, provided the original work is properly cited.

\begin{abstract}
Background: The recent data shows $16.55 \%$ prevalence of gestational diabetes mellitus in India. Hence universal screening has become important and we need a simple procedure which is economical and feasible to perform in the Indian context. The comparison of screening methods for gestational diabetes mellitus by 75 gm oral glucose challenge testing done in fasting state (WHO) and non-fasting (DIPSI) state was done.

Methods: Cross sectional prospective study done on 225 antenatal women between 20-24 weeks coming to tertiary level hospital in a single working unit were included. They were subjected to two screening glucose challenge tests for detection of gestational diabetes mellitus. Results of glucose challenge tests using $75 \mathrm{gm}$ of anhydrous glucose done in fasting and non fasting state were compared. Values of standard $100 \mathrm{gm}$ oral glucose tolerance test done were considered gold standard.

Results: The prevalence of gestational diabetes mellitus was found to be $12.4 \%$ by oral glucose tolerance testing. Glucose challenge test with $75 \mathrm{gm}$ done in fasting state was abnormal in $14.7 \%$ and abnormal in $15.6 \%$ done in non fasting state. The sensitivity of $75 \mathrm{gm}$ GCT done in fasting was $60.7 \%$ and the sensitivity of 75 gm GCT done in non fasting state was $67.9 \%$. The specificity of $75 \mathrm{gm}$ GCT done in fasting state was $91.9 \%$ and the specificity of 75 gm GCT done in non fasting state was $91.9 \%$.

Conclusions: Sensitivity and specificity of the screening tests done by 75 gm GCT done in non fasting state is comparable with screening done in fasting state. Screening done by $75 \mathrm{gm}$ GCT done in non fasting state is convenient and more feasible in Indian setup.
\end{abstract}

Keywords: Gestational diabetes mellitus, Glucose challenge test

\section{INTRODUCTION}

Gestational diabetes mellitus (GDM) is defined as "carbohydrate intolerance with recognition or onset during pregnancy, irrespective of the treatment with diet or insulin". ${ }^{1-4}$ The importance of gestational diabetes mellitus is that two generations are at risk of developing diabetes in the future. Women with a history of gestational diabetes mellitus are at increased risk of future diabetes, predominately type- 2 diabetes, as are their children. ${ }^{5}$ Besides, any abnormal glucose intolerance in pregnant women without gestational diabetes mellitus is associated with a graded increase in adverse maternal and fetal outcomes.

However, for the detection and diagnosis of gestational diabetes mellitus, controversy concerning optimal strategy still continues. The American Diabetes Association (ADA) recommends two step procedures for screening and diagnosis of diabetes in selective population. Compared with selective screening, universal screening for gestational diabetes mellitus detects more 
cases and improves maternal and fetal prognosis. ${ }^{6,7}$ In the Indian context, screening is essential in all pregnant women as the Indian women have an eleven fold increased risk of developing glucose intolerance during pregnancy compared to Caucasian women. ${ }^{8}$ The recent data shows $16.55 \%$ prevalence of gestational diabetes mellitus in our country. ${ }^{9}$ Hence universal screening has become important in our country.

Majority of the world has accepted the importance of screening but there is no consensus till now. ACOG recommends $50 \mathrm{gm}$ glucose challenge test. According to the WHO 1999 criteria, diagnosis was based on a 2-hour venous plasma glucose value of $\geq 140 \mathrm{mg} / \mathrm{dl}(7.8 \mathrm{mmol} / \mathrm{l})$ done in the fasting state, and this is called 'WHO 1999 criteria'. ${ }^{10-13}$ According to the Diabetes In Pregnancy Study group India (DIPSI) criteria, diagnosis of gestational diabetes mellitus was based on a 2-hour venous plasma glucose $\geq 140 \mathrm{mg} / \mathrm{dl}(7.8 \mathrm{mmol} / \mathrm{l})$ in the non-fasting OGTT, and this is called 'DIPSI criteria'. ${ }^{14}$

WHO predicts adverse pregnancy outcome and warrants treatment based on diagnosis of gestational diabetes mellitus by OGTT. A fasting plasma glucose value of $<95 \mathrm{mg} / \mathrm{dl}$ and a post 2-hour plasma glucose value after giving 75 gm glucose $\geq 140 \mathrm{mg} / \mathrm{dl}$ serves as screening as well as diagnostic criteria. It is a simple and economical one step procedure. ${ }^{13}$

Considering the magnitude of adverse pregnancy outcomes related to gestational diabetes the present study was undertaken to find out the prevalence of gestational diabetes mellitus in our our population using two glucose challenge tests.

The study also aimed to compare results of challenge test done by DIPSI criteria (non-fasting state) with that done by WHO criteria (fasting state) to ascertain whether the of sensitivity of screening done using by the guidelines recommended by Diabetes In Pregnancy Study Group India (DIPSI) is comparable to that done by WHO criterion.

\section{METHODS}

This was a cross-sectional prospective study carried in a single working unit of Lokmanya Tilak Municipal General Hospital, a major tertiary institute and Medical College.

Two twenty five antenatal women fulfilling inclusion criteria and who underwent all necessary tests of the study and finally delivered at our institute were analysed in this study from November 2015 to November 2017.

Approval of Institutional ethical committee was obtained before starting of the study. All patients recruited in the study were informed about the purpose and methodology of the study and informed consent was taken.

\section{Inclusion criteria}

Women with singleton pregnancy with gestational age between 20-24 weeks; women ready to participate; women willing to follow up and deliver at our institute.

\section{Exclusion criteria}

Women with overt diabetes mellitus; women on drugs affecting carbohydrate metabolism; women who do not tolerate glucose, who vomited glucose during the procedure; women with diagnosed multiple pregnancy; women with high risk factors for gestational diabetes mellitus like: age >25, family history of DM, BMI >30; and women who did not complete all tests.

After taking an informed consent, a standardized questionnaire was used to collect details.

Most women in our hospital report to antenatal clinics in the non-fasting state. A non-fasting OGCT using $75 \mathrm{gm}$ of anhydrous glucose administered irrespective of the timing of the last meal was carried out. A venous blood sample was drawn 2 hours after administering $75 \mathrm{gm}$ glucose.

After 3 days, patient was again called, this time in fasting state and again OGCT with 75 gm glucose load was done and blood sample was collected 2 hours later.

With a proper diet and overnight fasting, the patient was called the following week. A fasting sample was drawn and a $100 \mathrm{gm}$ oral glucose load was given followed by samples taken at intervals of 1 hour, 2 hours, 3 hours.

Patients not completing 3 tests visits for GCT/OGTT for reasons like non-compliance or intolerance, vomiting were excluded.

\section{Criteria used}

According to the WHO 1999 criteria, screening was based on a 2-hour venous plasma glucose value of $\geq 140$ $\mathrm{mg} / \mathrm{dl}(7.8 \mathrm{mmol} / \mathrm{l})$ done in the fasting state after $75 \mathrm{gm}$ oral glucose load, and this is called 'WHO 1999 criteria' for the purpose of my study.

According to the DIPSI criteria, national guidelines for diagnosis and management of gestational diabetes mellitus given by the Maternal Health Division, Ministry of Health and Family Welfare, Government of India, December 2014, screening of gestational diabetes mellitus was based on a 2-hour venous plasma glucose $\geq 140 \mathrm{mg} / \mathrm{dl}(7.8 \mathrm{mmol} / \mathrm{l})$ in the non-fasting state after 75 gm oral glucose load, and this is called 'DIPSI criteria' for the purpose of this study.

Gold standard are values of 100 gm OGTT done after patient reporting in fasting state. Carpenter and Coustan criteria was used. That is, if any 2 values meet or exceed 
FPG $>95 \mathrm{mg} / \mathrm{dl}, 1$ hour $\mathrm{PG}>180 \mathrm{mg} / \mathrm{dl}, 2$ hour PG $>155$ $\mathrm{mg} / \mathrm{dl}$ and 3 hour $\mathrm{PG}>140 \mathrm{mg} / \mathrm{dl}$ done in fasting state after a $100 \mathrm{gm}$ glucose load and this is the gold standard for the purpose of this study.

When any two of 4 values come abnormal, patient was labelled gestational diabetes mellitus.

Women diagnosed as gestational diabetes mellitus with abnormal OGTT/GCT were managed as per unit protocol till delivery. Though not a primary objectives of this study, since all these women finally delivered at our institute, their maternal, labour and perinatal outcome available from indoor sheets was analyzed.

\section{Statistical analysis}

All values were expressed as the mean \pm SD. Statistical analysis was performed using SPSS software (version 20) and MedCalc (version 12.7.0). Receiver operating characteristic curves were plotted using sensitivity and specificity for different non-fasting 2-hour venous plasma glucose values against the WHO and the DIPSI criteria. OGTT values were used as the gold standard, and the C statistic was calculated.

The information collected was recorded in a master chart in Microsoft Excel 2010 and analyzed using the social sciences software (SPSS) version 20.0. The quantitative data was represented as frequency or percentage, bar diagrams and pie charts. The association between two qualitative data was analyzed by Chi Square test and the 'p' value less than 0.05 was considered significant.

\section{RESULTS}

This was a prospective study of 225 pregnant women with single live intrauterine pregnancy between 20 to 24 weeks of gestation who eventually delivered at our hospital. A total of 225 subjects underwent both screening tests and OGTT and then were included in the final analysis to compare the screening methods for gestational diabetes mellitus by $75 \mathrm{gm}$ oral glucose challenge testing done in fasting state and non-fasting (random) state to assess the prevalence of gestational diabetes mellitus during the study period in low risk cases. The following are the results obtained from our study.

Patients in this study belonged to age group of 19 to 25 years with mean age 22.84 years with a standard deviation of 1.6. Out of the total 225 patients enrolled in this study, 89 were primigravida and 136 were multigravida. Majority of the patients had a normal BMI. Fourteen women were underweight and while 31 were overweight. The mean gestational age at time of screening was $22.52 \pm 1.00$ weeks with a range of $20-24.5$ weeks.
Screening of gestational diabetes mellitus done by $75 \mathrm{gm}$ test in non-fasting state (DIPSI criteria 2014) showed abnormal values in $15.6 \%$.

Value was considered abnormal (Screen Positive) when a 2-hour venous plasma glucose level was $\geq 140 \mathrm{mg} / \mathrm{dl}$ (7.8 $\mathrm{mmol} / \mathrm{l}$ ) after $75 \mathrm{gm}$ oral glucose load given to woman coming in the non-fasting state, and this was called 'DIPSI criteria' for the purpose of this study.

Table 1: Results of GCT done in the non fasting state (DIPSI criteria, 2014).

\begin{tabular}{|lll|}
\hline GCT & Frequency & Percentage \\
\hline Abnormal & 35 & 15.6 \\
\hline Normal & 192 & 84.4 \\
\hline Total & 225 & 100.0 \\
\hline
\end{tabular}

Abnormal OGCT values as per GCT done in fasting state (WHO criteria 1999) were seen in $14.7 \%$.

Screening test was considered positive or abnormal when 2 -hour venous plasma glucose value was $\geq 140 \mathrm{mg} / \mathrm{dl}$ $(7.8 \mathrm{mmol} / \mathrm{l})$ done after $75 \mathrm{gm}$ oral glucose load given to woman coming in the fasting state, and this was called 'WHO 1999 criteria' for the purpose of this study.

Table 2: Result of GCT done in the fasting state (WHO 1999 criteria).

\begin{tabular}{|lll|}
\hline GCT (fasting) & Frequency & Percentage \\
\hline Abnormal & 33 & 14.7 \\
\hline Normal & 192 & 85.3 \\
\hline Total & 225 & 100.0 \\
\hline
\end{tabular}

Standard 100 gm OGTT done after patient reporting in fasting state was considered as gold standard for diagnosis of gestational diabetes mellitus in our study. Carpenter and Coustan criteria was used. According to Carpenter and Coustan criteria, if any 2 values meet or exceed FPG 95 mg/dl, 1 hour PG $180 \mathrm{mg} / \mathrm{dl}, 2$ hours PG $155 \mathrm{mg} / \mathrm{dl}$ and 3 hours PG $140 \mathrm{mg} / \mathrm{dl}$ done in fasting state after a 100 gm glucose load, OGTT was considered abnormal. This is the gold standard for the purpose of this study.

When any two of 4 values came abnormal, patient was labelled gestational diabetes mellitus.

\section{Table 3: Abnormal $100 \mathrm{gm}$ OGTT at different intervals.}

\begin{tabular}{|lll|}
\hline Interval & Frequency & Percentage \\
\hline Fasting & 25 & 11.1 \\
\hline $\mathbf{1}$ hour & 27 & 12.0 \\
\hline $\mathbf{2}$ hour & 17 & 7.6 \\
\hline 3 hour & 8 & 3.6 \\
\hline
\end{tabular}


Table 4: Prevalence of gestational diabetes mellitus based on OGTT.

\begin{tabular}{|lll|}
\hline $\begin{array}{l}\text { Gestational diabetes } \\
\text { mellitus }\end{array}$ & Frequency & Percentage \\
\hline Present & 28 & 12.4 \\
\hline Absent & 197 & 87.6 \\
\hline Total & 225 & 100.0 \\
\hline
\end{tabular}

Prevalence of gestational diabetes mellitus based on abnormal GCT values as per OGTT criteria was $12.4 \%$.

When results of screening glucose challenge tests done in non-fasting (DIPSI) and fasting (WHO) were analysed compared to that by gold standard 100 gm glucose tolerance tests, sensitivity of challenge tests done in nonfasting (DIPSI) and fasting (WHO) states were comparable.

Table 5: Sensitivity and specificity of GCT done in fasting state (WHO criteria 1999) and non fasting state (DIPSI criteria, 2014).

\begin{tabular}{|lll|}
\hline & Sensitivity & Specificity \\
\hline Non fasting state & $67.9 \%$ & $91.9 \%$ \\
\hline Fasting state & $60.7 \%$ & $91.9 \%$ \\
\hline
\end{tabular}

Table 6: Mean venous plasma glucose values (mg/dl).

\begin{tabular}{|lllll|}
\hline & Minimum & Maximum & Mean & $\begin{array}{l}\text { Std. } \\
\text { deviation }\end{array}$ \\
\hline $\begin{array}{l}\text { 75 gm } \\
\text { GCT } \\
\text { fasting }\end{array}$ & 80 & 212 & 129.04 & 15.779 \\
\hline $\begin{array}{l}\text { 75 gm } \\
\text { GCT } \\
\text { random }\end{array}$ & 102 & 243 & 130.86 & 19.483 \\
\hline $\begin{array}{l}\text { OGTT 1- } \\
\text { hr }\end{array}$ & 101 & 313 & 161.60 & 22.139 \\
\hline $\begin{array}{l}\text { OGTT 2- } \\
\text { hr }\end{array}$ & 91 & 198 & 136.65 & 16.588 \\
\hline $\begin{array}{l}\text { OGTT 3- } \\
\text { hr }\end{array}$ & 77 & 151 & 121.77 & 13.834 \\
\hline
\end{tabular}

On analysing the delivery data, out of all the cases, 197 cases had delivered after completing 36 weeks of gestation.

Minimum gestational age at delivery was 31.1, maximum age was 41.5 in my study.

Out of the total deliveries, 169 were vaginal and 56 were abdominal.

Caesarean sections were done in $39.3 \%$ of the cases having gestational diabetes mellitus while it was in $22.9 \%$ in those who all didn't have gestational diabetes mellitus.

\section{DISCUSSION}

India is considered as capital of diabetes mellitus. Incidence of gestational diabetes mellitus is very high which affects adversely pregnancy outcome. Hence universal screening of gestational diabetes mellitus is recommended.

In developing countries such as India, particularly in rural areas, there are several challenges to screening for GDM. Some of these challenges include lack of trained phlebotomists, lack of standardized laboratories to do blood glucose estimations, and the problem in getting all women to visit in a fasting state. Due to these challenges, the WHO 1999 criteria, which require only a single sample (compared to three samples with the IADPSG and four samples with the Carpenter and Coustan criteria), became very popular in India. ${ }^{15}$ DIPSI also endorsed the 1999 WHO criteria and recommended universal screening at first contact and again at 24-28 weeks using this single-step 2-hour value, which the WHO (1999) criteria proposed. Because there are difficulties in getting women to visit in a fasting state for the OGTT, Anjalakshi et al conducted a study comparing the GTT done in the fasting and the nonfasting states. ${ }^{16}$ They found that the nonfasting OGTT had $100 \%$ specificity and sensitivity when compared to the fasting test taken as a "gold standard." Based on this study, DIPSI adopted the nonfasting OGTT as a single-step screening and diagnostic test for GDM in India. The DIPSI guidelines recommend using $75 \mathrm{~g}$ glucose load, which can be given in either a nonfasting or a fasting state, and one blood sample to be drawn 2 hours after glucose load, and a cutpoint of $140 \mathrm{mg} / \mathrm{dl}$ as the diagnostic cut-point for GDM irrespective of whether the GTT is done in the fasting or nonfasting state. ${ }^{17}$

In our study, we estimated the 2-hour plasma glucose after $75 \mathrm{gm}$ GCT without regard to the time of the last meal. All women also underwent WHO 2-hour PG after 75 gm GCT with overnight fasting. We found non-fasting GCT identified women with gestational diabetes mellitus similar to that of fasting GCT. Plasma glucose for each subject in non-fasting GCT and fasting GCT varied, yet all the values were found to be above the diagnostic criteria of 2-hour PG $140 \mathrm{mg} / \mathrm{dl}$. At the same time, majority of the women who were diagnosed to be non glucose tolerant by non-fasting GCT were found to have NGT by fasting GCT too. Their plasma glucose also varied but was above $140 \mathrm{mg} / \mathrm{dl}$. The non-fasting 2 hours post-75 gm glucose concentration strongly predicts adverse outcome for the mother and her offspring. ${ }^{12}$ The $75 \mathrm{gm}$ of glucose challenge though larger than the $50 \mathrm{gm}$ recommended by ADA, the difference in the glycemic load is not expected to result in a higher glycemic excursion in NGT subjects. ${ }^{16}$ Further, ADA also permits both 100 and $75 \mathrm{gm}$ OGTT for diagnosis of gestational diabetes mellitus. Though the glucose loads are different, the cut off values (FPG C95 mg/dl, 1-hour PG C180 $\mathrm{mg} / \mathrm{dl}$, 2-hour PG $\mathrm{C} 155 \mathrm{mg} / \mathrm{dl}$ ) for diagnosis of 
gestational diabetes mellitus are the same implying that the quantity of glucose load has little influence on the PG levels in a normal person, whereas in a metabolically deranged state like gestational diabetes mellitus, both 50 and $75 \mathrm{gm}$ glucose load would unmask the glucose intolerance.

Overall the present study showed that, DIPSI procedure, (2014) of 2-hour PG $\geq 140 \mathrm{mg} / \mathrm{dl}$ would be cost-effective without compromising the clinical equipoise.

Considering limited resources and logistic feasibility in our country, routine GCT done in antenatal women for screening of gestational diabetes mellitus in non-fasting state is acceptable option and can be recommended in our set of population. 75-gm GCT performed irrespective of the last meal timing is a patient-friendly approach. Women found to have non glucose tolerance in the first visit may need to undergo GCT later in the pregnancy. This one-step screening/diagnostic procedure is easy to perform, cost effective and causes least disturbance in a pregnant woman's routine activities.

\section{CONCLUSION}

The prevalence of gestational diabetes mellitus was found to be $12.4 \%$ in our study.

Therefore, universal screening for glucose intolerance during pregnancy is recommended in our ethnically vulnerable population with increased risk of developing gestational diabetes mellitus.

Sensitivity and specificity of the screening tests done by 75 gm GCT done in non fasting state is comparable with screening done in fasting state.

Screening done by 75 gm GCT done in non fasting state is more convenient and can be easily done in Indian setup where ANC women mostly attend OPDs in afternoon and in non fasting state. Asking women to come again in fasting state for such screening is likely to have low compliance.

Routine GCT done in antenatal women for screening of gestational diabetes mellitus in non-fasting state is acceptable option and can be recommended in our set of population considering limited resources and logistic feasibility in our country.

Funding: No funding sources

Conflict of interest: None declared

Ethical approval: The study was approved by the Institutional Ethics Committee

\section{REFERENCES}

1. Chan JCN, Malik V, Jia W, Kadowaki T, Yajnik CS, Yoon KH, et al. Diabetes in Asia: epidemiology, risk factors, and pathophysiology. JAMA. 2009;301(20):2129-40.

2. Danaei G, Finucane MM, Lu Y, Singh GM, Cowan MJ, Paciorek CJ, et al. National, regional, and global trends in fasting plasma glucose and diabetes prevalence since 1980: Systematic analysis of health examination surveys and epidemiological studies with 370 country-years and 2.7 million participants. Lancet. 2011;378(9785):31-40.

3. Olokoba AB, Obateru OA, Olokoba LB. Type 2 diabetes mellitus: a review of current trends. Oman Med J. 2012;27:269-73.

4. Balsells M, Garcia-Patterson A, Gich I, Corcoy R. Maternal and fetal outcome in women with type 2 versus type 1 diabetes mellitus: a systematic review and metaanalysis. J Clin Endocrinol Metab. 2009;94(11):4284-91.

5. Damm P. Gestational diabetes mellitus and subsequent development of overt diabetes mellitus. Dan Med Bull. 1998;45(5):495-509.

6. Xiong X, Saunders LD, Wang FL, Demianczuk NN. Gestational diabetes mellitus: prevalence, risk factors, maternal and infant outcomes. Int J Gynecol Obstet. 2001;75(3):221-8.

7. President T. Abstracts of the 48th EASD Annual Meeting of the European Association for the Study of Diabetes. Diabetologia. 2012;55:1-538.

8. Dornhorst A, Rossi M. Risk and prevention of type 2 diabetes in women with gestational diabetes. Diabetes Care. 1998;21(2):B43-9.

9. Seshlah V, Sahay BK, Das AK, Shah S, Banerjee S, Rao PV, et al. Gestational diabetes mellitus- Indian guidelines. J Indian Med Assoc. 2009;107(11):799806.

10. American Diabetes Association. Standards of medical care in diabetes-2009. Diabetes Care. 2009;32(1):S13-61.

11. Perkins JM, Dunn JP, Jagasia SM. Perspectives in gestational diabetes mellitus: a review of screening, diagnosis, and treatment. Clin Diabetes. 2007;25(2):57-62.

12. Metzger BE, Buchanan TA, Coustan DR, De Leiva A, Dunger DB, Hadden DR, et al. Summary and recommendations of the Fifth International Workshop-Conference on Gestational Diabetes Mellitus. Diabetes Care. 2007;30(2).

13. Alberti KG, Zimmet PZ. Definition, diagnosis and classification of diabetes mellitus and its complications. Part 1: diagnosis and classification of diabetes mellitus provisional report of a WHO consultation. Diabetes Med. 1998;15(7):539-53.

14. Mohan V, Mahalakshmi MM, Bhavadharini B, Maheswari K, Kalaiyarasi G, Anjana RM, et al. Comparison of screening for gestational diabetes mellitus by oral glucose tolerance tests done in the non-fasting (random) and fasting states. Acta Diabetol. 2014;51(6):1007-13.

15. Pettitt DJ, Bennett PH, Hanson RL, Narayan KMV, Knowler WC. Comparison of World Health Organization and National Diabetes Data Group 
procedures to detect abnormalities of glucose tolerance during pregnancy. Diabetes Care. 1994;17(11):1264-8.

16. Pettitt DJ, Bennett PH, Saad MF, Charles MA, Nelson RG, Knowler WC. Abnormal glucose tolerance during pregnancy in Pima Indian women. Long-term effects on offspring. Diabetes. 1991;40(2):126-30.

17. Anjalakshi C, Balaji V, Balaji MS, Ashalata S, Suganthi S, Arthi T, et al. A single test procedure to diagnose gestational diabetes mellitus. Acta Diabetol. 2009;46(1):51-4.

Cite this article as: Ardhapurkar SS, Kore SJ, Supe PA. Screening of gestational diabetes mellitus: comparison of glucose challenge test done in non fasting state (DIPSI criteria) and fasting state (WHO criteria): a prospective study. Int J Reprod Contracept Obstet Gynecol 2020;9:4918-23. 